Let $\mathrm{X}=\left(B C^{\prime}, B^{\prime} C^{\prime \prime}\right), \quad Y=\left(A C, A^{\prime} C^{\prime}\right), \quad \mathrm{Z}=\left(A B, A^{\prime} B^{\prime}\right), \quad B^{\prime \prime}$ $=\left(A C, O B^{\prime}\right), X^{\prime \prime}=\left(B C, B^{\prime \prime} C^{\prime}\right), Z^{\prime \prime}=\left(A B, A^{\prime} B^{\prime \prime}\right)$; thell, by Lemma 4 applied to the triangles $A B C$ and $A^{\prime} B^{\prime \prime} C^{\prime}$, the points $X^{\prime \prime} Y Z^{\prime \prime}$ are collinear. Also $Z A B Z^{\prime \prime}$ projects from $A^{\prime}$ into $B^{\prime} O B B^{\prime \prime}$, which projects from $C^{\prime \prime}$ into $X C B X^{\prime \prime}$, and hence $Z X, A C, Z^{\prime \prime} X^{\prime \prime}$ are concurrent in $Y$, that is, $X Y Z$ are collinear.

P'rinceton, New Jersey.

\title{
On a Chain of Circle Theorems.
}

By L. M. Brown.

If $P_{1}, P_{2}, P_{3}, P_{4}$ are four points on a circle $C$, and $P_{234}$ is the orthocentre of triangle $P_{2} P_{3} P_{4}, P_{134}$ the orthocentre of triangle $P_{1} P_{3} P_{4}$ and so on, then the quadrilateral $P_{234} P_{134} P_{124} P_{123}$ is congruent to the quadrilateral $P_{1} P_{2} P_{3} P_{4}$. This theorem seems to be due to Steiner (Ges. Werke, 1, p. 128; see H. F. Baker, Introduction to Plane Geometry, 1943, p. 332) and has appeared frequently since in collections of riders on the elementary circle theorems.

It is clear that $P_{234} P_{134} P_{124} P_{123}$ lie on a circle $C_{1234}$ equal to the original circle $C$. But also angle $P_{3} P_{134} P_{4}=P_{4} P_{1} P_{3}=P_{4} P_{2} P_{3}=$ $P_{3} P_{234} P_{4}$ (with angles directed and equations modulo $\pi$ ), and hence $P_{3} P_{4} P_{134} P_{234}$ lie on a circle $C_{34}$ equal to $C$, and which is in fact the mirror image of $C$ in $P_{3} P_{4}$. Similarly we obtain circles $C_{12}, C_{13}, C_{14}$, $C_{23}, C_{24}$, so that we have in all eight circles with four points on each. If any one of these be taken as the original circle, the same system of eight circles is obtained; if, e.g., we begin with $P_{3} P_{4} P_{134} P_{234}$ on the circle $C_{34}$, the four orthocentres are $P_{1}, P_{2}, P_{123}, P_{124}$ lying on $C_{12}$ and the remaining circles are the images of $C_{34}$ in the six sides of the quadrangle $P_{3} P_{4} P_{134} P_{234}$. Call this configuration $K_{4}$.

Let us now take a fifth point $P_{5}$ on $C$. Then any four of $P_{1} P_{2} P_{3} P_{4} P_{5}$ give a $K_{4}$. We have in fact five points $P_{.1} \ldots P_{5}$, ten points $P_{123} \ldots P_{345}$, a circle $C$, ten circles $C_{12} \ldots C_{45}$ and five circles $C_{1234} \ldots C_{2345}$. Then the circles $C_{1234} C_{1235} C_{1245} C_{1345} C_{2345}$ all pass through a point $P_{12345}$, completing a system of 16 points and 16 circles, five points on each circle and five circles through each point. We may show this by taking the circle $C_{12}$, e.g., on which lie the five points $P_{1} P_{2} P_{123} P_{124} P_{125}$ and build up the $K_{4}$ 's obtained by taking these four at a time. Use a parallel notation and write $Q_{1}=P_{1}, Q_{2}=P_{2}$, 
$Q_{3}=P_{123}, Q_{4}=P_{124}, Q_{5}=P_{125}$. Then by picking out orthocentres we obtain $Q_{123}=P_{3}\left(\right.$ i.e., the orthocentre of $Q_{1} Q_{2} Q_{3}$ is $\left.P_{3}\right), Q_{124}=P_{4}$, $Q_{125}=P_{5} ; Q_{134}=P_{134}, Q_{135}=P_{135}$ down to $Q_{245}=P_{245} ;$ but the final $Q$ point $Q_{345}$ is not the final $P$ point $P_{345}$. Then picking out the circles through these points, $D=C_{12}, D_{12}=C, D_{13}=C_{13}, D_{14}=C_{14}$, $D_{15}=C_{15}, D_{23}=C_{23}, D_{24}=C_{24}, D_{25}=C_{25} ; D_{34}=C_{1234}, D_{35}=C_{1235}$, $D_{45}=C_{1245} ; \quad D_{1234}=C_{34}, \quad D_{1235}=C_{35}, \quad D_{1245}=C_{45}, \quad D_{1345}=C_{1345}$, $D_{2345}=C_{2345}$. $\quad$ Now through $Q_{345}$ go $D_{34} D_{35} D_{45} D_{1345} D_{2345} ;$ i.e. the circles $C_{2345} C_{1345} C_{1245} C_{1235} C_{1234}$ all pass through one point $Q_{345}=P_{12345}$ as required. It is clear that the figure obtained in this way is symmetrical; any one of the 16 circles may be taken as the original circle with the five points on it as the original points; call it $K_{5}$.

If we next take a sixth point $P_{6}$ on $C$ and form the six $K_{5}$ 's by taking five points from $P_{1} \ldots P_{6}$, we have six points $P_{1} \ldots P_{0}, 20$ points $P_{123} \ldots P_{456}$, and six points $P_{12345} \ldots P_{23456}$. These lie on a circle $C, 15$ circles $C_{12} \ldots C_{56}$, and 15 circles $C_{1234} \ldots C_{3456}$. Then the six points $P_{12345} \ldots P_{23456}$ lie on a circle $C_{123456}$. This completes a symmetrical $K_{6}$ of 32 points and 32 circles, six points on a circle and six circles through a point. A proof of the existence of $C_{123456}$ on very similiar lines to that given above for the existence of $P_{12345}$ may be readily supplied by the reader.

It is now obvious that a chain of theorems may be constructed, the addition of every point $P_{i}$ on $C$ involving the existence alternately of a point and a circle, so that where there are $n$ points $P_{i}$, there are in all $2^{n-1}$ points and $2^{n-1}$ circles.

We should link this chain of theorems with the long catenation begun by Wallace, de Longchamps and Clifford. (See Baker, l.c., pp. 337-344, and Richmond, Proc. Edin. Math. Soc. 2, 6 (1939), 78 where a further bibliography is given). The chain given here is not in the normal de Longehamps' chain, but is a special case of Richmond's extension. Consider for simplicity a $K_{4}$, and let a general line $l_{1}$ through $P_{1}$ cut $C_{12}$ in $H_{12}, C_{13}$ in $H_{13}, C_{14}$ in $H_{14}$; let $l_{2}=H_{12} P_{2}$, $l_{3}=H_{13} P_{3}, l_{4}=H_{14} P_{4}$; let $l_{2} l_{3}$ cut in $H_{23}, l_{2} l_{4}$ in $H_{24}, l_{3} l_{4}$ in $H_{34}$. Then angle $P_{4} H_{34} P_{3}=P_{4} H_{14} H_{12}+H_{12} H_{13} P_{3}=P_{4} P_{134} P_{1}+P_{1} P_{134} P_{3}=$ $P_{4} P_{134} P_{3}(\bmod \pi)$. Hence $H_{34}$ lies on $C_{34}$ and similarly $H_{23}$ lies on $C_{23}$ and $H_{24}$ on $C_{24}$. Then if $P_{1} P_{2} P_{3} P_{4}$ and $l_{1} l_{2} l_{3} l_{4}$ be taken as the base points and lines of Richmond's extension the configuration is obtained as the 4-point case of this.

The Royal Technical College, Glasgow. 